\title{
Motor Skills Training Enhances Lesion-Induced Structural Plasticity in the Motor Cortex of Adult Rats
}

\author{
Theresa A. Jones, ${ }^{1,2}$ Catherine J. Chu, ${ }^{1}$ Lucinda A. Grande, ${ }^{1}$ and Aurora D. Gregory ${ }^{1}$ \\ ${ }^{1}$ Department of Psychology, and ${ }^{2 N e u r o b i o l o g y}$ and Behavior Program, University of Washington, Seattle, \\ Washington 98195
}

To assess behavioral experience effects on synaptic plasticity after brain damage, the present study examined the effects of complex motor skills training (the acrobatic task) on synaptic changes in layer $\mathrm{V}$ of the motor cortex opposite unilateral damage to the forelimb sensorimotor cortex (FLsmc). Adult male rats were given lesions or sham operations followed by $28 \mathrm{~d}$ of training on the acrobatic task [acrobat condition (AC)]. As a motor activity control [motor control (MC)], lesion and sham animals were given simple repetitive exercise. Previously, FLsmc lesions and acrobatic training have independently been found to result in increases in synapse to neuron ratios in the intact motor cortex relative to controls, and both of these effects were replicated in the present study. In addition, acrobat training after lesions significantly increased layer $V$ synapses per neuron relative to sham-AC and lesion-MC rats. Thus, the combination of acrobatic training and lesions resulted in an

Recent findings have suggested that neocortical damage in adult animals may make connected cortical regions especially sensitive to behavioral changes (for review, see Jones et al., 1998). Unilateral damage to the forelimb representation area of the sensorimotor cortex (FLsmc) in adult rats results in structural changes in the motor cortex opposite the lesions that appear to be dependent, in part, on forelimb behavior. After the lesions, rats have impairments in the use of the forelimb opposite the lesion and develop a compensatory reliance on the ipsilateral forelimb (the "nonimpaired" limb) for postural support behaviors (Jones and Schallert, 1992a). In layer V of the motor cortex opposite the lesion, there are time-dependent increases in dendritic arborization (Jones and Schallert, 1992a), neuropil volume, synapse to neuron ratios (Jones et al., 1996), and perforated synapses and multiple synaptic boutons (MSBs; Jones, 1999). Dendritic arborization increases were prevented by restricting the movements of the nonimpaired forelimb after the lesions (Jones and Schallert, 1994). However, the dendritic effects were not reproduced in intact animals that were forced to rely on one forelimb, suggesting that the magnitude and/or time course of neuronal growth results

Received June 15, 1999; revised Sept. 2, 1999; accepted Sept. 7, 1999.

This work was supported by National Institutes of Health Grant MH56361. We are very grateful to Drs. Jeffrey Kleim, Anna Klintsova, and William Greenough for advice on the design of the acrobatic task, to Dr. Timothy Schallert, Scott Bury, and DeAnna Adkins for comments on this manuscript, and to Jun Kim for assistance in preliminary data analyses and graphics.

Correspondence should be addressed to Dr. Theresa A. Jones, Department of Psychology, Guthrie Hall, Campus Box 351525, University of Washington, Seattle, WA 98195. E-mail: tajones@u.washington.edu.

Copyright (C) 1999 Society for Neuroscience 0270-6474/99/1910153-11\$05.00/0 enhanced synaptogenic response. Synapse subtypes were also differentially affected by the conditions. Lesion-MC and sham-AC primarily had increases in the number of synapses per neuron formed by multiple synaptic boutons in comparison to sham-MC. In contrast, lesion-AC had increases in both multiple and single synapses. Multiple synaptic spines and perforated synapses were also differentially affected by training versus lesions. On tests of coordinated forelimb use, lesion-AC rats performed better than lesion-MC rats. In addition to supporting a link between behavioral experience and structural plasticity after brain damage, these findings suggest that adaptive neural plasticity may be enhanced using behavioral manipulations as "therapy."

Key words: rehabilitative training; behavioral compensation; recovery of function; motor cortex; synaptogenesis; multiple synaptic boutons; perforated synapses; motor learning

from an interaction between lesion-induced central changes and behavioral changes.

A transient reduction in axonal processes is found in the motor cortex at time points preceding the neuronal growth, presumably a result of degeneration of transcallosal axons arising from the contralateral lesion site (Jones, 1999). Axonal degeneration has been found to result in increases in neurotrophic factors (for review, see Isackson, 1995; Ebadi et al., 1997) and cell adhesion molecules (Cotman et al., 1998), cytoskeletal restructuring (Sheppard et al., 1993; Hayes et al., 1995), and reactive changes in glia (Kelley and Steward, 1997; Ridet et al., 1997). These changes, which are likely to support the structural reorganization associated with reactive synaptogenesis, potentially make a region especially malleable in response to behaviorally driven changes in neuronal activity. If unilateral FLsmc lesions cause the opposite motor cortex to become more sensitive to behavioral changes, then it should be possible to capitalize on this sensitivity by using behavioral manipulations to enhance the structural changes.

The present study assessed the effects of complex motor skills training after unilateral FLsmc lesions on synaptic changes in layer V of the opposite motor cortex. Beginning after surgery, lesion or sham-operated male rats received $28 \mathrm{~d}$ of training on the acrobatic task, adapted from Black et al. (1990), which requires rats to learn a variety of coordinated movements to traverse a series of obstacles. As a motor activity control, rats received locomotor exercise requiring only simple repetitive movements. Acrobatic training has previously been found to result in an increase in synapse number per neuron in layer II/III of the motor cortex of intact adult female rats in comparison to motor controls (Kleim et al., 1996). The question of primary interest was 

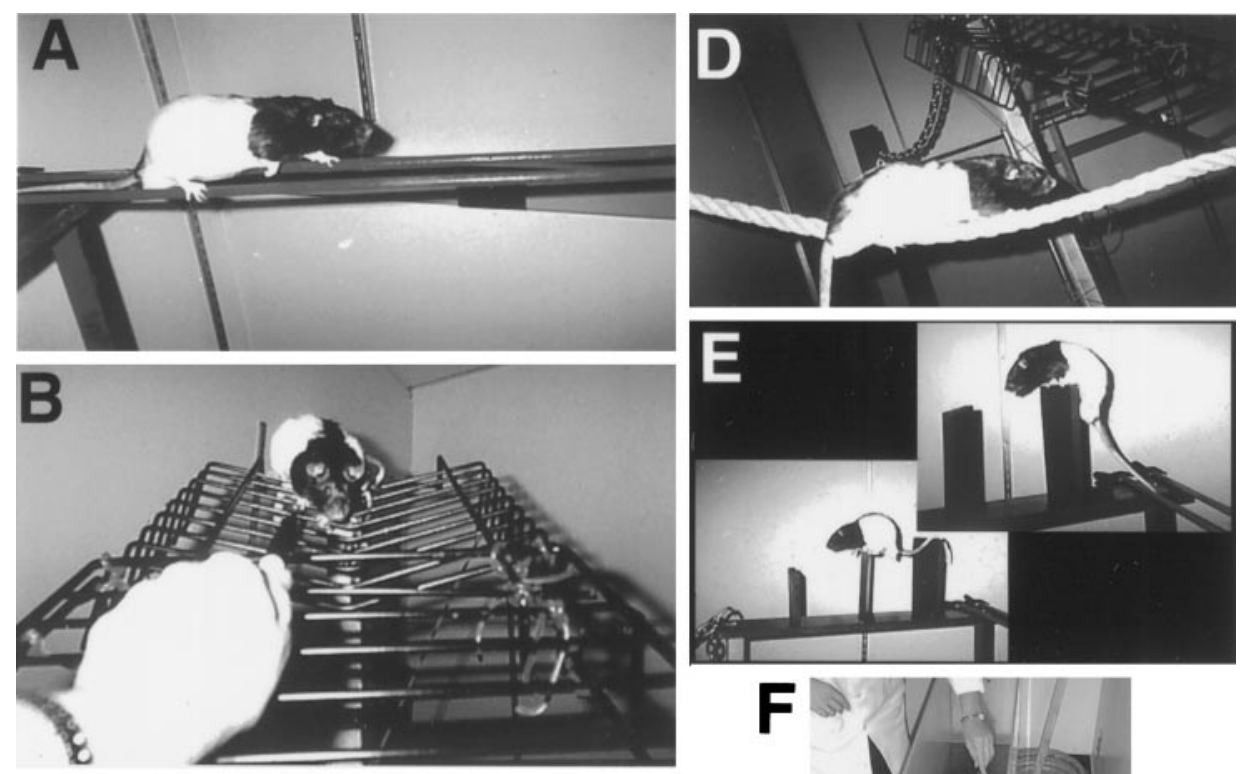

Figure 1. Rats trained on the acrobatic task were required to traverse a series of eight obstacles, which included small parallel rods $(A)$, a ladder with widely spaced rungs $(B)$, a grid platform $(C)$, a rope $(D)$, and barriers $(E)$. As a motor activity control, rats received simple repetitive exercise in a straight runway $(F)$.
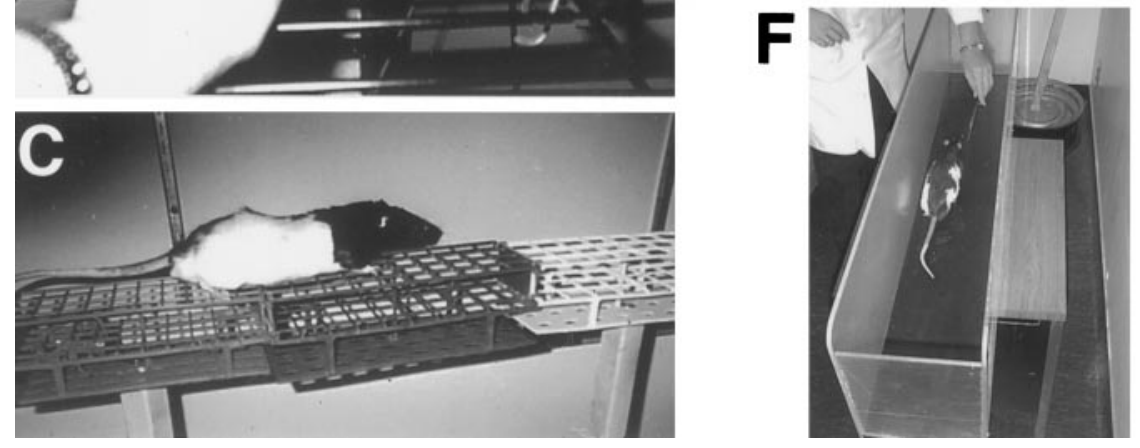

whether motor skills training after unilateral FLsmc lesions would enhance, or otherwise alter, the synaptic changes found as a result of either the lesion or the motor skills training alone.

\section{MATERIALS AND METHODS}

\section{Subjects and surgeries}

Forty male Long-Evans hooded rats between the ages of 4 and 5 months of age were used. Rats were made tame by frequent handling beginning after weaning and were housed in groups of two in transparent tub cages, receiving food and water ad libitum. Rats were randomly assigned to four groups: (1) rats receiving unilateral FLsmc lesions and postoperative training on the acrobatic task [lesion-acrobatic condition (AC); $n=11$ ], (2) rats with unilateral FLsmc lesions receiving postoperative simple repetitive exercise as a motor activity control [lesion-motor control (MC); $n=9$ ], (3) sham-operated rats receiving acrobatic task training (sham-AC; $n=10$ ), and (4) sham-operated motor control rats (sham$\mathrm{MC} ; n=10)$.

Surgical methods. Rats were anesthetized with Equithesin $(150 \mathrm{mg} / \mathrm{kg}$ chloral hydrate and $34 \mathrm{mg} / \mathrm{kg}$ pentobarbital). Unilateral lesions were aimed at the FLsmc, which includes the overlapping primary somaticsensory (SI) and primary motor (MI) representations of the forelimb, as described by Hall and Lindholm (1974). Skull was removed between 0.5 $\mathrm{mm}$ posterior to $1.5 \mathrm{~mm}$ anterior and $3.0-4.0 \mathrm{~mm}$ lateral to midline. After removal of dura, an uninsulated 30 gauge platinum wire electrode was lowered $1.7 \mathrm{~mm}$ below dura and slowly moved in 8 equally spaced traverses through the exposed cortex as $1 \mathrm{~mA}$ anodal constant current was delivered for $2 \mathrm{~min}$. Movement of the electrode and current delivery were continuous over the $2 \mathrm{~min}$. These lesions were chosen because they have previously been found to result in synaptic structural changes and increases in synapse number per neuron in the contralateral cortex (Jones et al., 1996; Jones, 1999) and thus provided a convenient model in which to assess skills training effects on lesion-induced synaptic changes. In previous examinations using cytoarchitectonics to identify sensorimotor cortical regions, these lesion procedures have been found to produce complete or near complete damage to the overlapping SI and MI area (the "overlap zone") of the forelimb as well as considerable damage to nonoverlapping SI and MI forelimb representational areas (Barth et al.,
1990; Jones and Schallert, 1992b). These lesions also result in moderate atrophy of the ipsilateral striatum and ventrobasal thalamus and neuron loss in the ipsilateral substantia nigra pars reticulata (Jones and Schallert, 1992b). Sham-operated rats received all procedures up to, but not including, removal of the skull (skull removal was not performed in sham operates because it has been found to produce behavioral and neurochemical asymmetries) (Adams et al., 1994). Atropine sulfate $(0.1 \mathrm{mg} /$ $\mathrm{kg}$ ) was used preoperatively and postoperatively to counteract respiratory depressive effects of Equithesin.

\section{Behavioral methods}

Motor skills training. Rats received postoperative training on an elevated obstacle course, the acrobatic task (Fig. 1), adapted from Black et al. (1990) (see also Kleim et al., 1996). This task requires rats to learn a variety of skilled forelimb, hindlimb, and coordinated whole-body movements. In this first test of the question of whether motor skills training after FLsmc lesions may alter synaptic changes in the motor cortex, this complex task was chosen in favor of more subtle manipulations of limb use, such as reach training, to maximize the ability to determine whether motor skills training affects lesion-induced structural changes in the motor cortex. It does not permit the determination of which components of the acrobatic training are necessary for these effects.

Acrobatic rats were required to sequentially traverse a rod $(2.7 \mathrm{~cm}$ diameter), rope (2 cm diameter), a ladder with $5 \mathrm{~cm}$ spaced rungs, a heavy metal link chain $(2.7 \times 4.3 \mathrm{~cm}$ links), barriers (13- to 21 -cm-high), small parallel rods $(1.1 \mathrm{~cm}$ diameter each), a grid platform $(85 \times 11 \mathrm{~cm}$ with $6.25,8.4$, and $12.25 \mathrm{~cm}^{2}$ openings), and a chasm leading to a flat platform. Between 2 and $4 \mathrm{~d}$ after surgery, rats received light training on the acrobatic task consisting of exposure to the testing room and placement on the first obstacle of the task (day 2), one heavily aided (by the experimenter) traverse of the task (day 3 ), and two aided traverses of the task (day 4). Subsequently, rats received full training consisting of four trials per day (days 5-13) followed by two trials per day (days 14-28). Rats were guided through the task by following the end of a marker or experimenter's hand and, occasionally, by light prodding applied to hindquarters. Other motivation for rapid and skillful task completion were the return to the home cage and the avoidance of falling and postural instability, respectively. Most rats required occasional assistance 
on the task, such as minor postural adjustments made by the experimenter, for the first several days of full training. The time (latency) to complete the task was measured on days $6-28$. Latency has been reported to be a useful measure of acrobatic task performance because it is sensitive to both the errors and hesitancy to traverse the obstacles (Kleim et al., 1996). Latency data per each $2 \mathrm{~d}$ of training were pooled to simplify the data analysis. In addition, forelimb and hindlimb foot slips were measured on days 11,19 , and 28 .

Motor control rats were yoked to acrobatic rats and were required to run back and forth in a simple straight alley $(1.2 \mathrm{~m} \times 20 \mathrm{~cm})$ for the duration of each acrobatic trial. When necessary, rats were prompted to run in the alley by chasing a pen or experimenter's hand and by occasional light prodding. The simple repetitive movements required in this control group were not intended to reproduce the motor movements of the acrobatic group. Instead, this group provides a control for the effects of locomotor exercise, exposure to the testing room, and handling. Early in the training, 10 rats displayed symptoms of adynamic ileus (colonic pseudo-obstruction) that was traced to one batch of Equithesin. These rats were dropped from the study (and not included in $n$ values above) before the completion of behavioral training.

Measures of forelimb use asymmetries. To assess whether acrobatic training influences lesion-induced asymmetries in forelimb use for postural support during exploratory movements, this behavior was measured before surgery ("day 0") and on several postsurgical days. Rats were videotaped for $2 \mathrm{~min}$ in a transparent cage. Instances of the use of a single forelimb and simultaneous use of both forelimbs for upright support against the cage walls were recorded during slow-motion playbacks by experimenters blind to the experimental conditions. Single forelimb use was considered to be any instance of support with one forelimb when the other forelimb was not in physical contact with the cage wall. These observations have previously been found to reveal forelimb asymmetries after sensorimotor cortical lesions (Jones and Schallert, 1992a, 1994; Kozlowski et al., 1996; Kawamata et al., 1997; Schallert et al., 1997) and do not require manipulations that may confound behavioral experience effects. Furthermore, these behaviors are very frequently observed and easily quantified and appear to represent important motor behaviors displayed by the rats whenever they are active (Jones and Schallert, 1992a). Percentage of ipsilateral forelimb use was calculated as the number of observations of ipsilateral forelimb use divided by the sum of total wall support observations (ipsilateral, contralateral, and simultaneous bilateral support $) \times 100$.

Tests of sensorimotor function. On day 29 , rats were administered two tests to assess acrobatic training effects on postlesion sensorimotor function. The Simultaneous Bilateral Tactile Stimulation Test measures the responsiveness to tactile stimulation applied to the distal forelimbs (Schallert et al., 1983). Asymmetries on this test have been found to be independent of postural-motor asymmetries and may be largely insensitive to practice and other experience effects (Schallert and Whishaw, 1984; Rose et al., 1987). Thus, in the present study, this test may be primarily sensitive to differences in the severity of the lesion. For this test, rats were removed briefly from their home cages to apply adhesive tape (Avery self-adhesive removable labels; $1.3 \mathrm{~cm}^{2}$ area) to both forelimbs. Rats were returned to their cages, and the order (left vs right) and latency to contact the stimuli were recorded for four or five trials. The footfault test was performed to assess whether acrobatic training enhanced coordinated placement of the forelimbs (Barth et al., 1990). Rats were placed on an elevated grid platform $(33 \times 30 \mathrm{~cm})$ for 2 min. Rats moved across the platform by placing their paws on the rungs of the grid. Errors were measured as slips with either forelimb through the grid openings. Although the configuration of the grid and task parameters were different, the grid openings used for the footfault test were identical to those used for the grid obstacle of the acrobatic task. The possibility that the exposure to these tests on the day before perfusions contributed to structural changes in the motor cortex cannot be ruled out. However, because all groups received these tests, any effects of this exposure would be expected to be equivalent between groups.

\section{Histological methods}

On postsurgical day 30, rats were anesthetized with a lethal dose of sodium pentobarbital and perfused intracardially with $0.1 \mathrm{M}$ phosphate buffer followed by $2 \%$ paraformaldehyde (depolymerized to formaldehyde) and $2.5 \%$ glutaraldehyde in the same buffer $\left(\mathrm{pH} 7.4-7.5 ; 21-23^{\circ} \mathrm{C}\right.$; all solutions). This time point was chosen because increases in motor cortical synapses per neuron have previously been found at $30 \mathrm{~d}$ after FLsmc lesions to the opposite hemisphere (Jones et al., 1996). Brains were extracted and stored overnight in fixative. Alternating sets of 100 and $300 \mu \mathrm{m}$ coronal sections of the cerebrum were obtained using a vibratome. For lesion hemispheres, sets of $100 \mu \mathrm{m}$ coronal sections were stained with methylene blue-azure II and used to verify lesion extent. Cytoarchitectonics was used to delineate damage to sensorimotor cortical subregions. For the hemisphere opposite the lesion and sham procedure (randomly assigned at the time of surgery), samples of the motor cortex were prepared for quantification of neuronal and synaptic density. A stereomicroscope with reflected light was used to locate the overlapping region of the forelimb primary motor and primary somatic sensory cortex in unstained $300 \mu \mathrm{m}$ sections. As described previously (Jones et al., 1996), the overlap zone of the forelimb is discernible in unstained coronal sections based on laminar differences in opacity and the distinct cytoarchitectural characteristics of this region. The agranular cortex medial to the overlap region was presumed to contain the forelimb motor representation area, based on previous combined cytoarchitectural and electrophysiological studies (Wise and Donoghue, 1986). Approximately 2-mm-wide samples from this region were removed between bregma and $1.2 \mathrm{~mm}$ anterior to bregma. Samples were fixed using buffered osmium tetroxide, stained en bloc with $2 \%$ uranyl acetate, dehydrated, and sandwich-embedded in Eponate 12 resin using standard resin embedding procedures. Serial coronal semithin $(0.8 \mu \mathrm{m})$ and ultrathin sections were obtained from the samples using an Ultracut $\mathrm{R}$ microtome (Leica, Nussloch, Germany). Semithin sections were stained with toluidine blue and used for Layer V neuronal density estimates. Serial ultrathin $(70 \mathrm{~nm})$ sections of layer $\mathrm{V}$, used for electron microscopy, were mounted on slotted grids and stained with lead citrate. These sections were cut at a microtome setting of $70 \mathrm{~nm}$, showed silver-gray interference colors, and were assumed to be $70 \mathrm{~nm}$ in thickness. To ensure consistency between groups in procedures that may affect tissue shrinkage and section thickness, tissue from all groups was processed together for each step of histological processing. All light- and electron-microscopic measurements were made on tissue that was coded to conceal experimental condition.

\section{Stereological methods}

The present investigation was concerned with changes in synapse number and structure in the motor cortex opposite the lesion and in shams. Because cortical volume can change, measures of synaptic density may not accurately reflect changes in synapse number. Increases in the thickness and volume of the cortex have been found in many studies of experience effects on cortical structure (Rosenzweig et al., 1962; Turner and Greenough, 1985) (for review, see Greenough and Black, 1992), and increases in motor cortical volume have previously been found after lesions of the opposite FLsmc (Jones and Schallert, 1992a; Jones et al., 1996). Neuronal density decreases when cortical volume increases and, thus, neuronal density measures can be used to assess changes in cortical volume, avoiding the error-prone delineation of the boundaries of cortical subregions. Estimates of the number of synapses per neuron accurately reflect changes in synapse number when neuron number is stable (Anker and Cragg, 1974). In previous work, volume increases in the cortex opposite FLsmc lesions were found to be similar in magnitude to neuronal density decreases (Jones and Schallert, 1992a; Jones et al., 1996) suggesting that, if there is a loss of neurons resulting from the lesions, this loss is subdetectable. Thus, in the present study, measurement of synapse number per neuron was chosen as the most sensitive method of detecting net changes in layer $\mathrm{V}$ synapse number. Because measurements of neuronal and synaptic density were obtained from the same resin-embedded samples, tissue shrinkage effects contributed equally to synaptic density and neuronal density data and, therefore, were unlikely to bias synapse number per neuron data.

Neuronal density measures. The density of layer V neurons in the motor cortex opposite the lesion (presumed forelimb region) was measured using the physical dissector method (Gundersen et al., 1988) with the aid of a computer-assisted light-microscopic stereological workstation and NIH Image software. In this method, neuronal nuclei that are present in one section are counted if they are not also present in an adjacent section. Neuronal nuclei were counted using every other section of 12 serial 0.8 $\mu \mathrm{m}$ sections at $785 \times$ magnification. Layer $\mathrm{V}$ was identified primarily by its characteristically large pyramidal neurons and by its position within the approximate middle one-third of layers II-VI of the motor cortex. Neurons were identified by multiple criterion including the presence of a central nucleolus, a pale nucleus surrounded by perikaryal cytoplasm and, frequently, by the oval to pyramidal morphology of the soma. Cells with characteristics of glia were not included in the analyses (i.e., cells 
Figure 2. Examples of synapses formed by MSBs $(A, B)$, a synapse formed by an MSS $(C)$, and synapses with perforated postsynaptic densities $(A, D)$ in layer $\mathrm{V}$ of the motor cortex. $A, B$, MSBs consist of a single axonal bouton forming synaptic contacts (arrows) with two or more postsynaptic dendritic processes, i.e., spines or shafts. One of the synapses in $A$ has a perforated postsynaptic density (double arrows). Synapses between individual pairings of boutons and spines, or single synapses, are indicated by open arrows. $C$, MSSs consist of a single dendritic spine forming synaptic contacts (arrows) with two or more axonal boutons. $D$, A synapse between a single synaptic spine and single synaptic bouton with a perforated postsynaptic density (arrows). Scale bar, $0.5 \mu \mathrm{m}$.
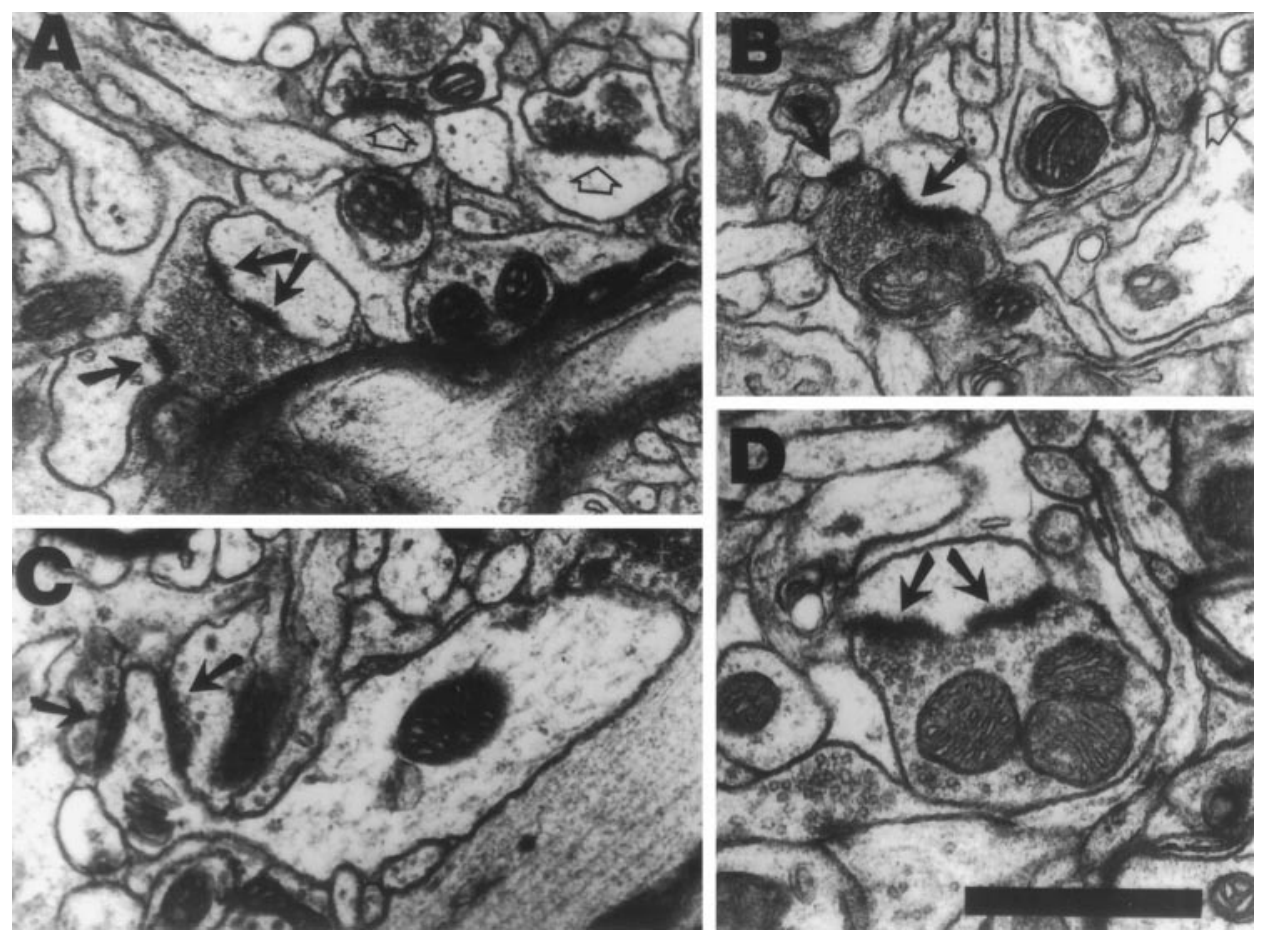

with clumping of chromatin centrally or adjacent to the nuclear membrane and, typically, small nuclei and sparse or irregularly shaped surrounding perikarya). Unbiased sample frames $\left(37,250 \mu \mathrm{m}^{2}\right.$ each) were positioned within layer $\mathrm{V}$ using a systematic random sampling scheme that included samples throughout the dorsal to ventral depth of layer V. Six sample frames were used per section for a total of 40 samples per animal. These data are reported as the layer $\mathrm{V}$ sample volume per neuron (the inverse of neuronal density).

Synaptic measures. Synaptic density was measured using the physical dissector method in two sets of eight serially positioned electron micrographs $(20,060 \times$ final magnification). Synapses appearing within an unbiased sample frame $\left(93 \mu \mathrm{m}^{2}\right)$ were counted only if they were not present in an adjacent section. Axodendritic synapses were identified by the presence of at least three vesicles in the presynaptic bouton and the presence of a postsynaptic density. Synaptic density was calculated as the number of synapses counted divided by the sample volume. For each animal, the number of layer V synapses per neuron was calculated as the product of synaptic density and layer $\mathrm{V}$ volume per neuron (note that this synapse to neuron ratio is not a measure of the number of synapses onto layer $\mathrm{V}$ neurons, given the intermingling of dendritic processes across cortical layers, but rather is a sensitive indication of net changes in synapse number within layer $\mathrm{V}$ ).

In addition to quantification of all axodendritic synapses, the density and number per neuron of synapses with perforated (or segmented) postsynaptic densities (PSDs) and synapses formed by MSBs and by multiple synaptic spines (MSSs) were obtained (Fig. 2).

MSB synapses consist of synapses formed between an individual axonal bouton and more than one dendritic processes (shafts or spines). MSS synapses are those formed between a spine and more than one axonal bouton. MSBs were chosen for quantification because they have previously been found to increase in number per neuron in the motor cortex opposite FLsmc lesions (Jones, 1999). They have also been found to increase in other brain areas in response to behavioral (Federmeier et al., 1994; Jones et al., 1997), physiological, and hormonal (Hatton, 1990; Woolley et al., 1996) changes. MSSs were chosen for quantification because it seemed reasonable to assess whether the multiple usage of synaptic elements applies to postsynaptic processes. Multiple synapses were counted using methods that provide accurate comparisons of experimental groups, as described previously (Jones et al., 1997; Jones, 1999). Synapses were classified as MSB or MSS synapses if an additional contact was formed by the bouton or spine, respectively, within three sections of the first synapse, moving in both directions through the set of serial sections. For MSSs, the multiple contacts could be on the head or neck of the spine, but the classification did not include branched spines.
Perforated synapses are those with an interrupted or completely partitioned PSD (Geinisman et al., 1996). These were chosen for quantification because they have previously been found to increase in the motor cortex after acrobatic training (Kleim et al., 1997; see also Keller et al., 1992). They have also been found to increase after several other types of behavioral manipulations (Greenough et al., 1978; Stewart and Rusakov, 1995) and have been linked to synaptic efficacy increases in hippocampus (Buchs and Muller, 1996; Geinisman et al., 1996). Synapses with two or more completely separate PSDs and those with one or more clearly distinguished interruptions in an otherwise continuous PSD were classified as perforated synapses. Synapses with a continuous PSD but irregularly shaped outer edges were not included in this conservative classification, as verified by following individual PSDs through the section series.

\section{Statistical analyses}

For each anatomical variable and for tests of functional recovery, planned comparisons of the following group effects were performed: (1) lesion-AC versus lesion-MC, (2) lesion-AC versus sham-AC (3) lesion-MC versus sham-MC and (4) sham-AC versus sham-MC. Planned comparisons were performed using SAS statistical analysis software for general linear model (GLM) procedures for contrasts (SAS Institute, 1988). Performance on the acrobatic task and limb use asymmetry were analyzed using SAS GLM procedure for two-way ANOVA for the effects of group by day. Preliminary analyses in sham-operated rats of ipsilateral versus contralateral forelimbs (sides assigned at the time of surgery) revealed no significant effects for any of the behavioral measures, and these data were pooled in subsequent analyses. Post hoc comparisons were performed, when appropriate, using SAS GLM procedures for contrasts.

\section{RESULTS}

\section{Characterization of lesion placement and extent}

Based on the extent of remaining non-necrotic tissue, all lesions appeared to produce major damage to the overlapping region of the forelimb sensory and motor representation areas. In many animals $(n=5$, lesion-AC; $n=5$, lesion-MC), there was no discernible remaining forelimb overlap zone. In the remaining animals, only small portions of the overlap zone could be seen extending from the lateral edges of the lesions, indicating that most of this region was damaged. All lesions also produced 
considerable damage to the lateral agranular cortex and granular cortex bordering the forelimb overlap zone (and presumed to contain the nonoverlapping MI and SI representations of the forelimb, respectively) (Wise and Donoghue, 1986). Approximately half of the animals in each group $(n=6$, lesion-AC; $n=$ 5 , lesion-MC) had slight damage to the medial agranular cortex. In addition, all but one animal (in the lesion-MC) had minor damage to the rostral extension of the hindlimb overlap zone. As is typical in tissue examined at relatively late time points after the lesions (Jones, 1999), there were deformations of adjacent cortex and underlying tissue that give the appearance of filling in the lesion site (Fig. $3 A$ ). Although there was variability in the medial to lateral dimensions of the damage within groups, there were no major differences in the extent of the lesions between groups, based on reconstructions of the extent of remaining non-necrotic tissue (Fig. 3B). Any region of the corpus callosum lacking a cover of cortical tissue was assumed to have sustained at least superficial damage. Given this criteria, nearly all lesions produced at least superficial damage to the corpus callosum $(n=9$, lesion-AC; $n=$ 8 , lesion-MC). Of these, three lesion-AC and two lesion-MC rats had lesions that clearly protruded into the depth of the corpus callosum in at least one section. One rat had a localized penetration of the corpus callosum and superficial damage to the dorsal striatum (lesion-AC group; Fig. 3B). There were no differences in the measured anatomical or behavioral data that could be traced to variations in lesion placement or extent, including the extent of callosal damage.

\section{Overall synaptic changes in the motor cortex}

As shown in Figure $4 A$, rats with unilateral FLsmc lesions receiving simple repetitive exercise as a motor activity control (lesion-MC) had a greater number of layer $\mathrm{V}$ synapses per neuron (i.e., synapse to neuron ratio) in the motor cortex opposite the lesion than sham-MC $\left(F_{(1,36)}=4.66 ; p<0.05\right)$. Similarly, acrobatic training in sham-operated rats (sham-AC) resulted in a significantly greater number of synapses per neuron in comparison to sham-MC $\left(F_{(1,36)}=4.96 ; p<0.05\right)$. When acrobatic training was performed after the lesions (lesion-AC), there was a major elevation in synapse number per neuron that exceeded that found as a result of motor skills training alone (lesion-AC vs sham-AC; $\left.F_{(1,36)}=5.88 ; p<0.05\right)$ and lesions alone (lesion-AC vs lesion-MC; $\left.F_{(1,36)}=5.61 ; p<0.02\right)$.

Increases in synapse number per neuron can occur as a result of an increase in the packing density of synapses and/or if synaptic density remains unchanged in an expanded volume of cortex. Although not significant relative to acrobatic and lesion controls, subtle elevations in both volume per neuron (Fig. 4B) and synaptic density (Fig. 4C) in lesion-AC combined to result in the significant synapse number per neuron increases. In comparison to sham-MC rats, a significantly greater volume per neuron was found in lesion-MC rats $\left(F_{(1,36)}=15.47 ; p<0.0005\right)$ and sham-AC rats $\left(F_{(1,36)}=12.74 ; p<0.001\right)$. There were no significant effects for synaptic density for any comparison.

\section{Synapses formed by multiple versus single synaptic boutons}

Lesion-AC was the only group to show a significant elevation in synapses formed by single synaptic boutons. In the absence of acrobatic training, unilateral FLsmc lesions failed to result in significant changes in the number of single synapses (Table 1), consistent with previous findings (Jones, 1999). Acrobatic training in sham rats also failed to result in significant changes in this

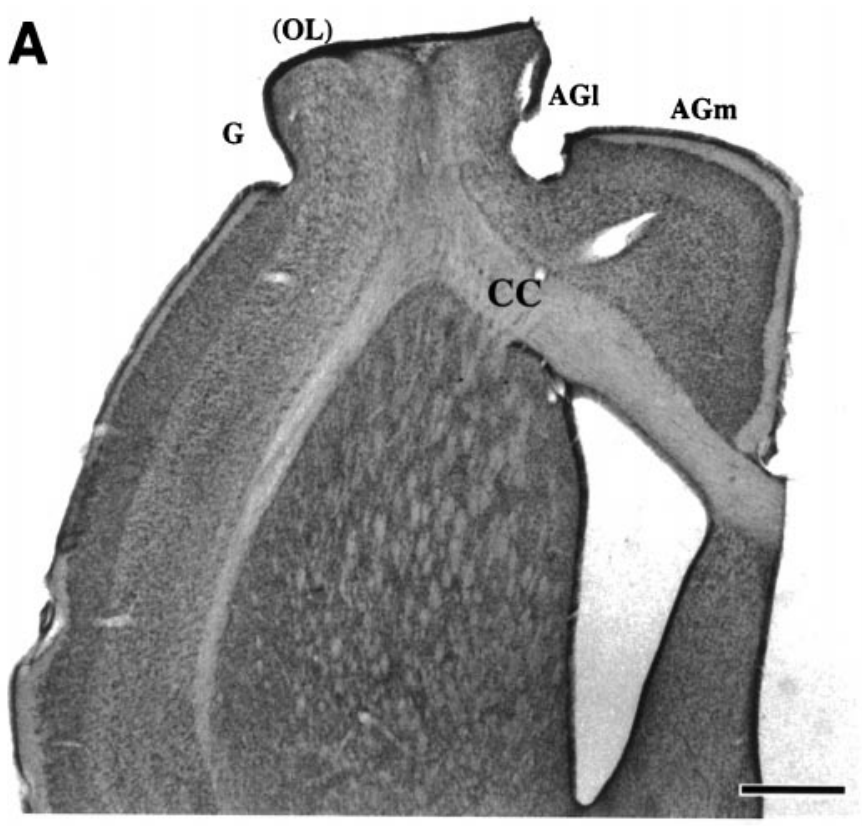

B

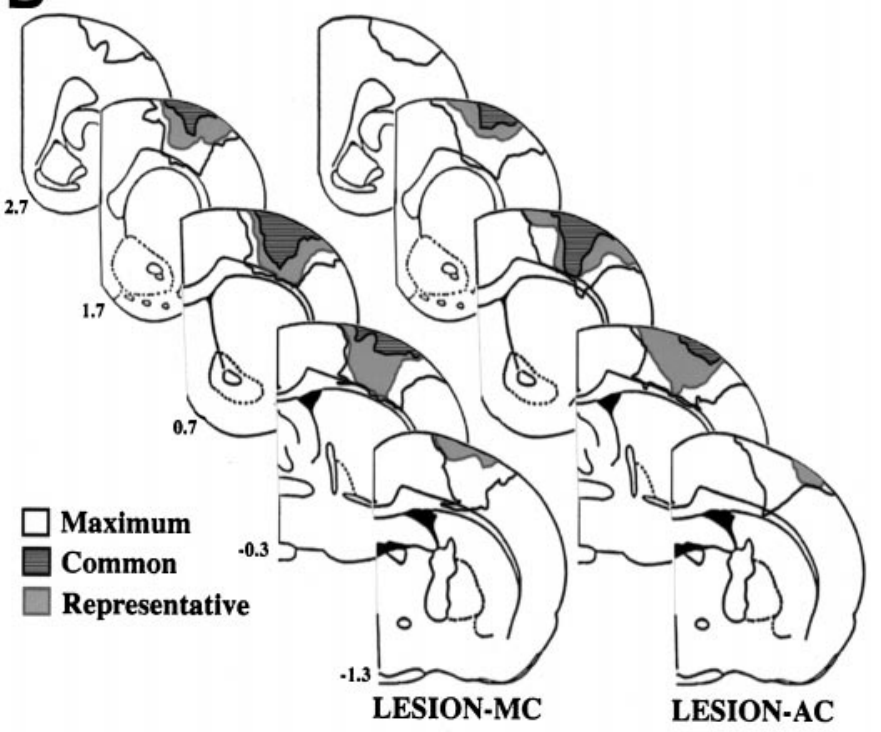

Figure 3. A, Photomicrograph of a representative FLsmc lesion in a coronal hemisection stained with methylene blue-azure II. Lesions created complete or near complete damage to the overlapping SI and MI representations of the forelimb $(O L)$ as well as extensive damage to the adjacent MI (within lateral agranular cortex, $A G l$ ) and SI (within granular cortex, $G$ ) cortex. In this example, there is no normal appearing overlap zone remaining. $A G m$, Medial agranular cortex; $C C$, corpus callosum. Scale bar, $1 \mathrm{~mm}$. $B$, Lesions reconstructed based on the extent of remaining non-necrotic tissue. The outer boundaries of all lesions combined (maximum), the region of damage common to all lesions (common), and a representative lesion (representative), for each lesion group are shown. Numbers to the left indicate coordinates relative to bregma. Structural measurements were made in the motor cortex opposite the lesions.

subtype of synapse (similar to the results of complex environment housing) (Jones et al., 1997). The number of single synapses in lesion-AC was significantly greater than sham-AC $\left(F_{(1,36)}=\right.$ 9.44; $p<0.005)$ and lesion-MC $\left(F_{(1,36)}=8.11 ; p<0.01\right)$.

Unlike the effects on single synaptic boutons, there was no further increase in synapses formed by MSBs in lesion-AC rats in 

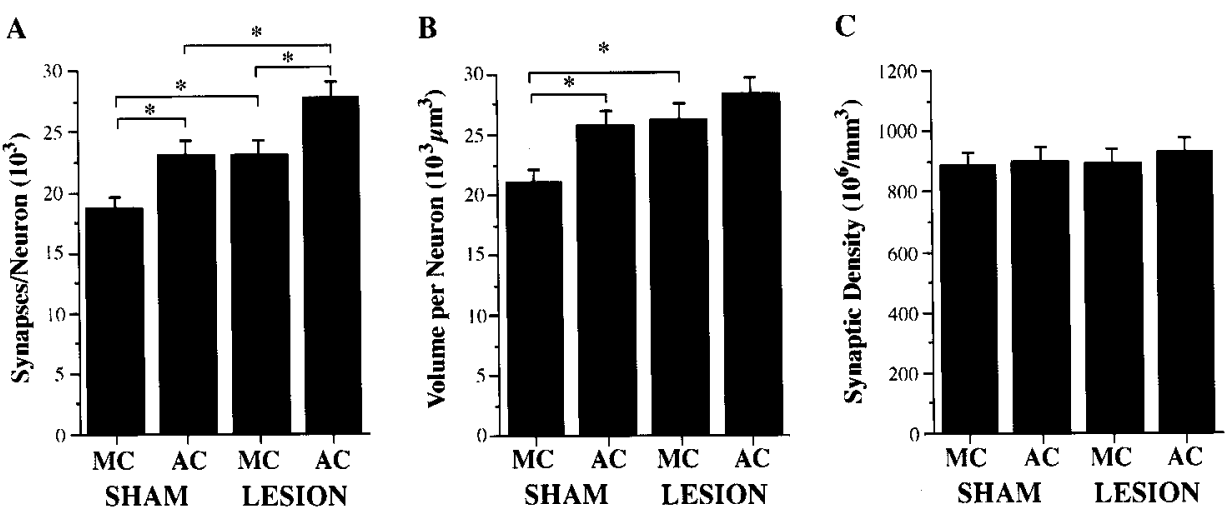

Figure 4. Structural effects in layer V of the motor cortex opposite unilateral FLsmc lesions or sham operations in AC and MC rats. $A$, Acrobatic training after lesions (lesion-AC) significantly enhanced synapse number per neuron increases in comparison to the increases that were found as a result of the training alone (sham-AC) or as a result of the lesion in motor control rats (lesion-MC). B, Volume per neuron (the inverse of neuronal density) was significantly increased in sham-AC and lesion-MC in comparison to sham-MC. Volume per neuron was elevated but not significantly increased in lesion-AC relative to sham-AC and lesion-MC. $C$, There were no significant differences in the density of layer $\mathrm{V}$ synapses for any planned comparison. ${ }^{*} p<0.05$.

\section{Table 1. Changes in single and multiple synaptic boutons}

\begin{tabular}{lll} 
& \multicolumn{2}{l}{ Synapses formed by } \\
\cline { 2 - 3 } & Single synaptic boutons & Multiple synaptic boutons \\
\hline Sham-MC & $16.56 \pm 0.92$ & $1.58 \pm 0.37$ \\
Sham-AC & $19.09 \pm 1.29$ & $2.87 \pm 0.42^{* *}$ \\
Lesion-MC & $19.30 \pm 0.94$ & $2.70 \pm 0.24$ \\
Lesion-AC & $23.64 \pm 1.06^{* * * *}$ & $2.92 \pm 0.48$
\end{tabular}

Data are $10^{3}$ layer $\mathrm{V}$ synapses per neuron (synaptic density/neuronal density). ${ }^{*} p<0.005$ significantly different from sham of the same training condition.

$* * p<0.05$ significantly different from motor control of the same lesion condition.

comparison to either sham-AC $\left(F_{(1,36)}=0.01 ; p>0.05\right)$ or lesion-MC $(F=0.14 ; p>0.05)$. Sham-AC had a significantly greater number of these synapses than sham-MC $\left(F_{(1,36)}=5.23\right.$; $p<0.05)$. Lesion-MC showed an elevation in MSB synapse number per neuron that approached significance in comparison to sham-MC $\left(F_{(1,36)}=3.76 ; p=0.06\right)$. There were no significant changes in the density (number per unit volume) of single or MSB synapses for any comparison. Thus, the overall increase in synapse number per neuron in lesion-AC rats was primarily a result of a disproportionate increase in synapses formed by single synaptic boutons.

Increases in MSB synapse number can result from their de novo formation and/or from a recruitment from the pre-existing pool of single synaptic boutons. If the latter predominated in the present study, then lesion-MC and sham-AC must add new single synaptic boutons (i.e., to replace those that have become MSBs). However, any addition of single synapses in these groups was not sufficient to significantly increase single synapse number per neuron.

\section{Synapses formed by single versus multiple synaptic spines}

As shown in Table 2, similar to the effects found for single synaptic boutons, the greatest number of single synaptic spines per neuron was found in lesion-AC rats. This increase was significant in comparison to sham-AC $\left(F_{(1,36)}=5.15 ; p<0.05\right)$. In comparison to lesion-MC, the greater number of single synaptic spines in lesion-AC approached significance $\left(F_{(1,36)}=3.90\right.$; $p=0.056)$.
Table 2. Changes in single and multiple synaptic spines

\begin{tabular}{lll} 
& \multicolumn{2}{l}{ Synapses formed by } \\
\cline { 2 - 3 } & Single synaptic spines & Multiple synaptic spines \\
\hline Sham-MC & $15.37 \pm 0.94$ & $0.85 \pm 0.14$ \\
Sham-AC & $17.89 \pm 1.44$ & $1.77 \pm 0.37^{*}$ \\
Lesion-MC & $18.29 \pm 0.72$ & $0.96 \pm 0.17$ \\
Lesion-AC & $21.70 \pm 1.44^{*}$ & $1.58 \pm 0.25$
\end{tabular}

Data are $10^{3}$ layer V synapses per neuron (synaptic density/neuronal density). ${ }^{*} p<0.05$ significantly different from motor control of the same lesion condition.

Table 3. Changes in perforated synapses

\begin{tabular}{lll} 
& All perforated synapses & Perforated MSB synapses \\
\hline Sham-MC & $1.27 \pm 0.17$ & $0.12 \pm 0.05$ \\
Sham-AC & $1.86 \pm 0.23$ & $0.26 \pm 0.06$ \\
Lesion-MC & $1.91 \pm 0.22^{*}$ & $0.40 \pm 0.12^{*}$ \\
Lesion-AC & $2.07 \pm 0.22$ & $0.27 \pm 0.05$
\end{tabular}

Data are $10^{3}$ layer $\mathrm{V}$ synapses per neuron (synaptic density/neuronal density). ${ }^{*} p<0.05$ significantly different from sham of the same training condition.

Acrobatic training in sham rats resulted in a significantly greater number of synapses per neuron formed by MSS synapses $\left(\right.$ sham-AC vs sham-MC; $\left.F_{(1,36)}=6.57 ; p<0.02\right)$. The density of MSS synapses was also significantly greater $\left(F_{(1,36)}=5.03 ; p<\right.$ $0.05)$ in sham-AC versus sham-MC. There was no further increase in MSS synapses in lesion-AC rats $\left(F_{(1,36)}=0.29 ; p>\right.$ $0.05)$. Furthermore, lesion-MC animals failed to show a significant change in MSS synapses relative to sham-MC $\left(F_{(1,36)}=\right.$ $0.08 ; p>0.05)$. These data indicate that MSS synapses are increased by acrobatic training but not by FLsmc lesions. There were no significant changes in the proportion of shaft versus spine synapses as a result of the lesions and/or training conditions.

\section{Synapses with perforated PSDs}

Table 3 shows the total number of synapses per neuron with perforated PSDs ("perforated synapses"). Unilateral lesions resulted in a significant elevation in the total number of perforated synapses per neuron in comparison to shams (lesion-MC vs sham-MC; $\left.F_{(1,36)}=4.36 ; p<0.05\right)$. Acrobatic training resulted in 

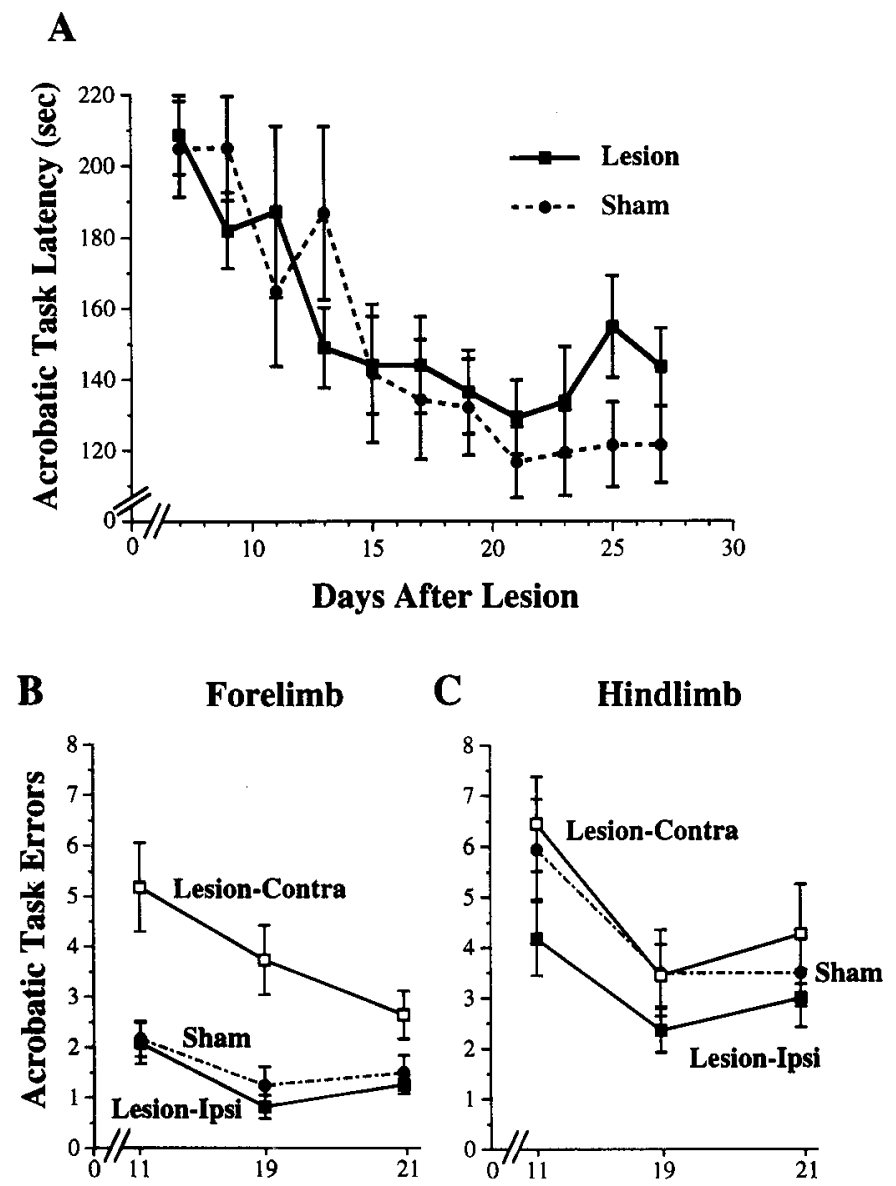

Days After Lesion

Figure 5. Performance on the acrobatic task in lesion-AC and sham-AC rats. $A$, Latency in seconds to complete the acrobatic task over days of training. There was a significant reduction in latency over days of training $(p<0.0001)$ but no significant differences between lesion and sham animals on this measure. The data shown are from the first traverse of the acrobatic task per training session. $B$, Forelimb errors in limb placement on the acrobatic task, measured as the number of foot slips per traverse. The lesions resulted in a significant increase in contralateral forelimb errors in comparison to sham $(p<0.0005)$. Ipsilateral forelimb errors were not significantly different from sham. $C$, There were no significant differences between lesion and sham rats in hindlimb footslips.

an elevation that approached significance (sham-AC vs sham$\left.\mathrm{MC} ; F_{(1,36)}=3.92 ; p=0.055\right)$. Acrobatic training after FLsmc lesions did not result in a significant increase in perforated synapses in comparison to sham-AC or lesion-MC $(F$ values $=0.48$ and 0.25 , respectively; $p$ values $>0.05)$.

Consistent with previous findings (Jones, 1999) FLsmc lesions resulted in a marked increase in perforated synapses formed by MSBs (lesion-MC vs sham-MC; $F_{(1,36)}=7.23 ; p<0.01$ ). In contrast, acrobatic training alone did not result in a significant change in this synapse subtype in comparison to sham-MC $(F=$ 1.66; $p>0.05)$. Lesion-AC rats were not significantly different in comparison to either sham-AC or lesion-MC. Together, these data suggest that there is no additive or other effect of acrobatic training after lesions on the prevalence of perforated PSDs.

\section{Performance on the acrobatic task}

Figure $5 A$ shows latencies to complete the acrobatic task (first trial per day). Lesion-AC and sham-AC groups increased in

speed over days of training and showed similar acquisition rates using this measure. Two-way ANOVA for the effects of group by day revealed a significant effect for day $\left(F_{(10,190)}=11.40 ; p<\right.$ $0.0001)$. There was no significant effect for group $\left(F_{(1,19)}=0.04\right.$; $p>0.05)$ and no significant group by day interaction $\left(F_{(10,190)}=\right.$ $1.31 ; p>0.05)$.

Figure $5 B$ shows errors in forelimb placements on the acrobatic task. Lesion-AC rats made more errors with the contralateral (to the lesion) forelimb in comparison to forelimb errors made by sham-AC rats. Contralateral forelimb errors were significantly increased in lesion-AC in comparison to sham-AC (two-way ANOVA effects for group, $\left.F_{(1,19)}=19.65 ; p<0.0005\right)$. There was also a significant effect for day $\left(F_{(2,38)}=4.54 ; p<0.02\right)$, but no significant group by day interaction $\left(F_{(2,38)}=1.51 ; p>0.05\right)$. Thus, although lesion-AC completed the task as quickly as shams, this was accomplished with less accuracy in the use of the impaired forelimb. For ipsilateral forelimb errors, although there was a significant effect for day $\left(F_{(2,38)}=7.81 ; p<0.002\right)$, there were no significant effects for group $\left(F_{(1,19)}=0.60 ; p>0.05\right)$ or group by day interaction $\left(F_{(2,38)}=0.19 ; p>0.05\right)$.

Figure $5 C$ shows errors in hindlimb placements on the acrobatic task. Although lesion-AC rats tended to make more contralateral than ipsilateral hindlimb errors, there were no significant group differences in errors made with either hindlimb $\left(F_{(1,19)}\right.$ $=1.73$ and 0.17 , ipsilateral and contralateral hindlimb, respectively; $p$ values $>0.05)$. There was a significant reduction in errors over days of training (days, $F_{(2,38)}=11.71$ and 11.31, ipsilateral and contralateral errors, respectively; $p$ values $<0.0001$ ), but no significant group by day interaction effects for either hindlimb $\left(F_{(2,38)}=0.89\right.$ and 0.23 , ipsilateral and contralateral hindlimb, respectively; $p$ values $>0.05)$.

\section{Asymmetry in the use of the forelimbs}

Figure 6 shows asymmetry in the use of the forelimbs for upright postural support behaviors. Unilateral FLsmc lesions resulted in preferential use of the forelimb ipsilateral to the lesion and a proportionate reduction in the use of the contralateral (impaired) forelimb. Acrobatic training did not significantly alter this asymmetrical use of the forelimbs. In two-way ANOVA, of the percentage of use of the ipsilateral forelimb, there was a significant effect for group $\left(F_{(3,37)}=7.01 ; p<0.001\right)$, day $\left(F_{(5,175)}=6.01\right.$; $p<0.0001)$, and group by day interaction $\left(F_{(15,175)}=2.11 ; p<\right.$ $0.02)$. Similarly, for percentage of use of the contralateral forelimb, there was a significant effect for group $\left(F_{(3,37)}=6.67 ; p<\right.$ $0.001)$, day $\left(F_{(5,175)}=3.97 ; p<0.002\right)$, and group by day interaction $\left(F_{(15,175)}=1.84 ; p<0.05\right)$. In both lesion groups, post hoc analyses revealed a significant elevation in use of the ipsilateral forelimb and a reduction in use of the contralateral forelimb in comparison to sham groups for most postsurgical days (Fig. 6). There were no significant differences in lesion-AC versus lesion-MC in the percentage of use of either forelimb for any day of observation. In addition, the simultaneous use of both forelimbs for upright support behaviors was not significantly reduced as a result of the lesion. There were no significant group, day, or interaction effects. Pooling across postoperative days, the mean \pm SEM percentage of simultaneous use of the forelimbs was $22.94 \pm$ 1.78 in lesion-AC, $24.70 \pm 2.02$ in lesion-MC, $26.72 \pm 1.51$ in sham-AC, and $29.48 \pm 1.49$ in sham-MC.

\section{Performance on tests of functional recovery}

Figure 7 shows performance on the footfault task, measured on day 29 after surgeries. In both sham and lesion groups, acrobatic 


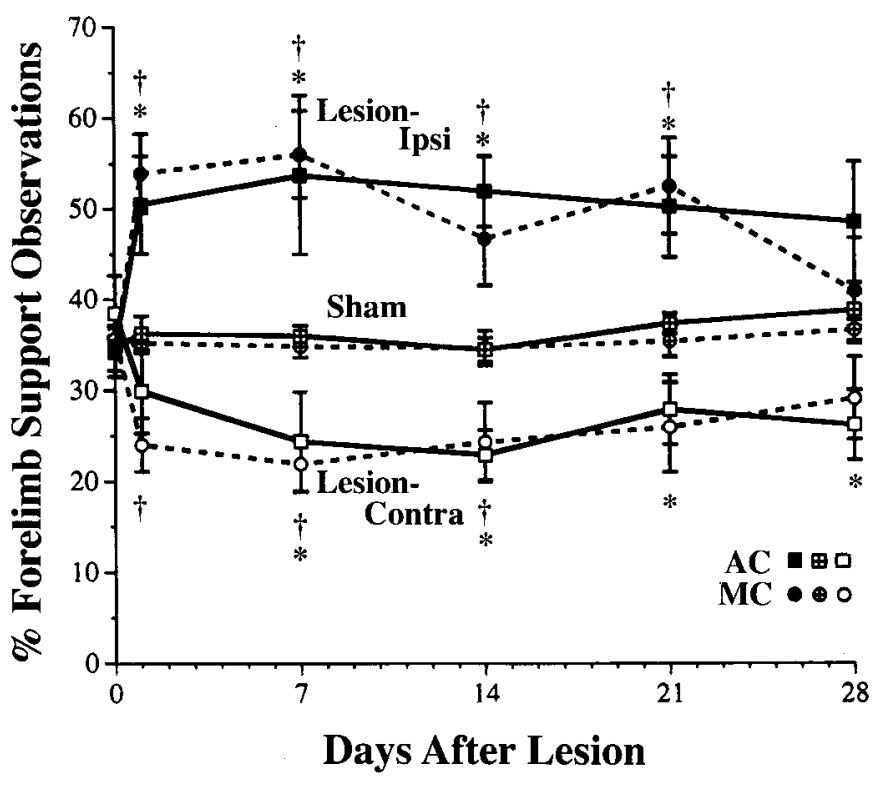

Figure 6. Forelimb asymmetries in postural support behaviors during exploratory movements. These data are from observations of the use of the forelimbs for upright postural support against the walls of a transparent cage. FLsmc lesions resulted in the preferential use of the forelimb ipsilateral to the lesion and a reduction in the use of the contralateral forelimb in comparison to shams. There were no significant differences between lesion-AC and lesion-MC in forelimb use asymmetries. Data for lesions are percentage ipsilateral or contralateral forelimb use/total support observations (ipsi + contra + bilateral support). For shams, data for left and right forelimbs are pooled. The percentage of simultaneous use of both forelimbs was not significantly affected by the lesions (see Results). ${ }^{*} p<0.05$ lesion-AC versus sham-AC; $\dagger p<0.05$ lesion-MC versus sham-MC.

training resulted in improvements in performance, as measured by a reduction in the number of forelimb errors (foot slips), in comparison to motor controls. In lesion-AC rats, both forelimbs were improved, but the improvements were notably greater in the ipsilateral than in the contralateral forelimb. In comparison to lesion-MC, lesion-AC rats made significantly fewer errors with both the ipsilateral forelimb $\left(F_{(1,36)}=17.47 ; p<0.0002\right)$ and with the contralateral forelimb $\left(F_{(1,36)}=9.20 ; p<0.005\right)$. Similarly, sham-AC rats made fewer errors than sham-MC $\left(F_{(1,36)}=11.16\right.$; $p<0.002)$. Both lesion groups made more errors with the contralateral forelimb in comparison to sham groups of the same training conditions (lesion-AC vs sham-AC, $F=9.65, p<0.005$; lesion-MC vs sham-MC, $F=5.10, p<0.05$ ).

Although lesion-AC made fewer errors with either forelimb in comparison to lesion-MC, the acrobatic training amplified the asymmetry in forelimb errors. In lesion-AC rats, the percentage of contralateral forelimb errors [\% contra errors/(contra + ipsi errors)] was $75.76 \pm 6.44$. In lesion-MC, the percentage of contralateral errors was $56.73 \pm 3.87$, which was significantly different from lesion-AC $\left(F_{(1,18)}=5.72 ; p<0.05\right)$.

In contrast to the training effects on the footfault task, performance on the bilateral tactile stimulation test was not affected by the acrobatic training. This test has been found to be independent of postural-motor asymmetries and experience effects (Schallert et al., 1983; Schallert and Whishaw, 1984; Rose et al., 1987) and thus, the results were expected to be primarily sensitive to differences in lesion severity rather than acrobatic training effects. Lesion animals in both training conditions showed moderate asymmetries in responding to the bilaterally applied stimuli, tend-

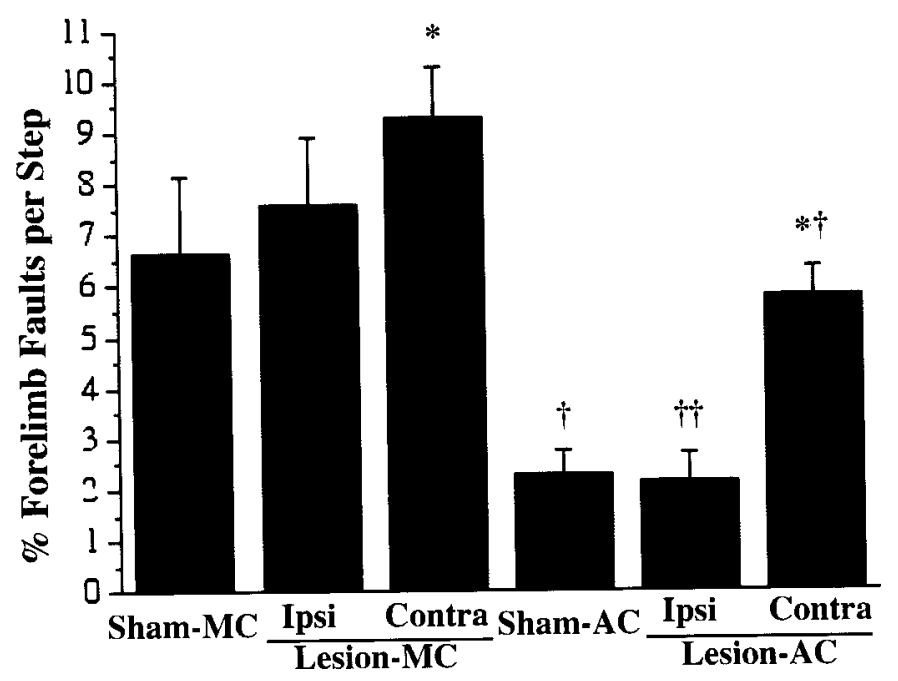

Figure 7. Performance on the footfault task. This task was performed after the last day of training (on postoperative day 29) as a test of coordinated forelimb placement during locomotion. Acrobatic training in both lesion and sham animals resulted in a reduction in forelimb-placing errors on this task in comparison to lesion and sham motor controls, respectively. Lesion-AC made fewer errors with either forelimb in comparison to lesion-MC, most notably with the ipsilateral forelimb. ${ }^{*} p<$ 0.05 significantly different from sham of the same training condition, $\downarrow p<$ 0.005 and $\dagger \uparrow p<0.0005$ significantly different from motor control of the same lesion condition.

ing to preferentially contact and remove the stimuli applied to the ipsilateral forelimb before the contralateral forelimb. The percentage of trials in which the ipsilateral stimulus was contacted first was $75.8 \pm 6.8$ in lesion-AC, $80.0 \pm 10.5$ in lesion-MC, $38.7 \pm 8.8$ in sham-AC, and $57.0 \pm 10.1$ in sham-MC. The lesion-AC group was significantly different from sham-AC $\left(F_{(1,36)}=8.84 ; p<0.01\right)$, and lesion-MC tended to show an increased asymmetry relative to sham-MC $\left(F_{(1,36)}=3.07 ; p=\right.$ $0.09)$. There were no significant differences between the two lesion groups or between the two sham groups in response asymmetries. There were also no significant differences in the latencies to contact the tactile stimuli for any comparison.

\section{DISCUSSION}

\section{Training effects on overall synaptic increases}

The major finding of this experiment was that synaptogenesis in layer $\mathrm{V}$ of the motor cortex opposite FLsmc lesions was enhanced by postinjury training on a complex motor skills task. Consistent with previous findings in female rats (Kleim et al., 1996), acrobatic training in sham-operated male rats (sham-AC) resulted in an increase in motor cortical synapses relative to sham-motor controls (sham-MC). In motor control rats with FLsmc lesions (lesion-MC), increases in synapses per neuron were found relative to sham-MC, similar to the effects of these lesions in rats with no postoperative behavioral manipulations (Jones et al., 1996). Previous work has suggested that this postlesion structural plasticity is related to forelimb behavioral changes that occur spontaneously after the lesions (Jones and Schallert, 1994). In the present study, acrobatic training after FLsmc lesions (lesion-AC) resulted in a further increase in synapse to neuron ratios in the motor cortex in comparison to lesion-MC and sham-AC. The present findings support the hypothesis that motor cortical structural plasticity can be enhanced using appropriate postoperative behavioral training. 


\section{Changes in synapse structure and configuration}

The overall increase in synapse number per neuron in lesion-AC rats was primarily a result of a disproportionate increase in "single synapses" (single synaptic spines and boutons) in comparison to the other groups. Synapses formed by MSBs were a major component of the synapse increases in lesion-MC and sham-AC rats, consistent with previous findings (Jones, 1999; Jones et al., 1997). Lesion-AC rats had no significant increase in MSBs relative to these groups but had an abundant increase in synapses formed by single synaptic boutons. Similarly, lesion-AC rats had an increase in single synaptic spines relative to the other groups and no further increase in MSSs. The addition of MSB synapses appears to be a common route of synapse addition in adult animals (Hatton, 1990; Federmeier et al., 1994; Woolley et al., 1996; Jones et al., 1997; Kleim et al., 1997 ), and this tendency to reuse boutons has been hypothesized to be a type of constraint on the formation of synaptic connections in adult animals (Jones et al., 1997). If so, then the increase in single synapses in lesion-AC represents a major liberation from this constraint. It may be that changes triggered by degeneration and excitotoxicity in combination with neural activity changes induced by postinjury acrobatic training are, together, a potent enough combination to promote a more major addition of boutons and spines. These findings also suggest that the combined effects of acrobatic training and cortical lesions are not merely additive in nature because additive effects would have resulted in further increases in multiple synapses in lesion-AC rats.

With the exception of increases in single synapses, the changes in lesion-AC relative to the other groups appear to reflect additive effects of the training and the lesion. Lesions, but not acrobatic training alone, resulted in an increase in perforated MSB synapses. Acrobatic training, but not lesions alone, resulted in an increase in MSSs. Lesion-AC rats had increases in both subtypes. The synapse subtypes examined in the present study have each been related to learning and/or changes in neuronal excitability. Perforated and MSB synapses have been found to increase in several models of experience-dependent brain changes (Greenough et al., 1978; Stewart and Rusakov, 1995; Jones et al., 1997; Kleim et al., 1997). Perforated synapses have been linked to synaptic efficacy increases in the hippocampus (Buchs and Muller, 1996; Geinisman et al., 1996). MSBs, and MSSs have also been implicated in changes in patterns of activity in different brain regions (Hatton, 1990; Bendre et al., 1995; Harris, 1995; Robinson and Kolb, 1997). However, the functional relevance of, e.g., a greater addition of neocortical perforated MSB versus MSS synapses remains to be understood.

\section{Training effects on behavioral function}

Acrobatic training enhanced function of the forelimbs as measured on the footfault test. In lesion-AC, performance improved in both forelimbs, but the most dramatic improvements were found in the ipsilateral rather than the contralateral (impaired) forelimb. Enhancing function of nonimpaired extremities in a manner that compensates for the impaired limb may improve net functional outcome. Schallert et al. (1997) have found that much of the measured recovery of the contralateral forelimb on the footfault task is dependent on the nonimpaired (ipsilateral) forelimb. Local anesthetization of the ipsilateral forelimb resulted in a complete reinstatement of contralateral forelimb impairments in FLsmc lesion rats demonstrating full recovery on this task when permitted to use both forelimbs normally.
The focus of the present study was on changes in undamaged motor cortex; however, the acrobatic task seems capable of influencing structural plasticity in regions of the lesion hemisphere as well as the intact hemisphere. This possibility is supported by studies on motor cortical map plasticity in which behavioral training was found to influence reorganization of perilesion cortical representation areas (Castro-Alamancos and Borrell, 1995; Nudo et al., 1996). Such changes, if they occur after acrobatic training, may contribute to the improvements in the impaired forelimb on the footfault task.

\section{Implications for rehabilitative treatment of brain injury}

It has been known for considerable time that manipulations of postinjury behavioral experience, such as complex environment housing (Rose et al., 1993), can improve functional recovery (for review, see Jones et al., 1998). The central processes underlying this improvement have been poorly understood. A major issue is whether postinjury training produces changes that are different from intact animals. For example, rehabilitative training might simply rely on learning-related processes which, while providing benefit relative to brain-injured controls, are indistinguishable from those in intact animals undergoing the same training. Findings by Kolb and Gibb (1991) supported this possibility. Complex environment housing in intact adult rats and in adult rats with bilateral frontal cortex lesions produced comparable increases in dendritic arborization in the occipital cortex. The lesions increased arborization in the sensorimotor cortex independent of the housing condition. Thus, in contrast to the present study, there were no additive or interactive structural effects of the behavioral manipulation and lesion within the same cortical region. It may be that examination of a region that has already been found to be sensitive to cortical lesion-induced behavioral changes has optimized the ability to detect these effects in the present study. Brain areas showing heightened structural plasticity in response to spontaneously occurring behavioral changes after brain damage might be particularly sensitive to training procedures that manipulate similar behaviors.

Recent findings have indicated that some types of behavioral experience early after the damage may be detrimental to functional outcome. Limb-restricting vests that forced rats to use the impaired forelimb during the first week after unilateral FLsmc lesions worsened the function of the impaired limb and increased the size of the lesion (Kozlowski et al., 1996; Humm et al., 1998). Because these effects were blocked by an NMDA receptor antagonist, they may result from a use-related exaggeration of excitotoxicity (Schallert et al., 1997). These findings suggest that rehabilitation efforts, perhaps especially those undertaken early after the injury, are made at the risk of worsening function. This poses a potential dilemma because there also may be sensitive windows for enhancing function. In the present study, behavioral training improved function of the impaired forelimb. Furthermore, there were no clear differences in the extent of damage to sensorimotor cortical subregions between the two groups and no differences in response asymmetries on the bilateral tactile stimulation test, which are likely to be sensitive to differences in lesion extent (Kozlowski et al., 1996). In contrast to forced-use procedures, the acrobatic training permitted rats to use whatever behavioral strategies came naturally to complete the task. It may be that rats naturally avoid the types of limb use that would exacerbate the damage or it may simply be that the net use of the impaired limb is much reduced during vulnerable time-periods in the acrobatic rats in comparison to rats wearing limb-restricting vests. These 
findings clearly indicate that it is possible to undertake rehabilitative efforts involving the use of the impaired forelimb without worsening function of this limb.

The present study found that complex motor skills training improved functional performance and enhanced synaptogenesis in comparison to simple repetitive exercise after the cortical lesions. These findings suggest that learning new motor skills, rather than simply increasing use, may be important to optimize adaptation to the damage. However, given the complexity of the experience of acrobatic rats, the issue of which motor skills may be most effectively targeted to improve function remains a topic for future investigation. Although much interest is focused on enhancing the function of impaired modalities after brain injury, targeting nonimpaired functions for enhancement in a manner that compensates for lost function is also of major importance. The present findings support the idea that cortical regions involved in behavioral compensation after injury to a connected region may be made exceptionally labile and responsive to behavioral change. This greater lability may provide a major route for enhancing functional outcome, provided that it is appropriately capitalized on and, perhaps, capitalized on at the right time after the injury.

\section{REFERENCES}

Adams FS, Schwarting RK, Huston JP (1994) Behavioral and neurochemical asymmetries following unilateral trephination of the rat skull: is this control operation always appropriate? Physiol Behav 55:947-952.

Anker RL, Cragg BG (1974) Estimation of the number of synapses in a volume of nervous tissue from counts in thin sections by electron microscopy. J Neurocytol 3:725-735.

Barth TM, Jones TA, Schallert T (1990) Functional subdivisions of the rat somatic sensorimotor cortex. Behav Brain Res 39:73-95.

Bendre AA, Swain RA, Wheeler BC, Greenough WT (1995) Augmentation of cerebellar responses to parallel fiber activation following skilled motor acquisition in rats. Soc Neurosci Abstr 21:444.

Black JE, Isaacs K, Anderson BJ, Alcantara AA, Greenough WT (1990) Learning causes synaptogenesis whereas motor activity causes angiogenesis, in cerebellar cortex of adult rats. Proc Natl Acad Sci USA 87:5568-5572.

Buchs P-A, Muller D (1996) Induction of long-term potentiation is associated with major ultrastructural changes of activated synapses. Proc Natl Acad Sci USA 93:8040-8045.

Castro-Alamancos MA, Borrell J (1995) Functional recovery of forelimb response capacity after forelimb primary motor cortex damage in the rat is due to the reorganization of adjacent areas of cortex. Neuroscience 68:793-805.

Cotman CW, Hailer NP, Pfister KK, Soltesz I, Schachner M (1998) Cell adhesion molecules in neural plasticity and pathology: similar mechanisms, distinct organizations? Prog Neurobiol 55:659-559.

Ebadi M, Bashir RM, Heidrick ML, Hamada FM, Refaey HE, Hamed A, Helal G, Baxi MD, Cerutis DR, Lassi NK (1997) Neurotrophins and their receptors in nerve injury and repair. Neurochem Int 30:347-374.

Federmeier K, Kleim JA, Anderson BJ, Greenough WT (1994) Formation of double synapses in the cerebellar cortex of the rat following motor learning. Soc Neurosci Abstr 20:1435.

Geinisman Y, deToledo-Morrell L, Morrell F, Persina IS, Beatty MA (1996) Synapse restructuring associated with the maintenance phase of hippocampal long-term potentiation. J Comp Neurol 368:413-423.

Gundersen HJG, Bendtsen TF, Korbo L, Marcussen N, Møller A, Nielsen K, Nyengaard JR, Pakkenberg B, Sørensen FB, Vesterby A, West MJ (1988) Some new, simple and efficient stereological methods and their use in pathological research and diagnosis. Acta Pathol Microbiol Immunol Scand 96:379-394.

Greenough WT, Black JE (1992) Induction of brain structure by experience: substrates for cognitive development. In: Developmental behavioral neuroscience, Vol 24 (Gunnar M, Nelson C, eds), pp 155-200. Hillsdale, NJ: Lawrence Erlbaum.

Greenough WT, West RW, DeVoogd TJ (1978) Subsynaptic plate perforations: changes with age and experience in the rat. Science 202:1096-1098.
Hall RD, Lindholm EP (1974) Organization of motor and somatosensory neocortex in the albino rat. Brain Res 66:23-38.

Harris KM (1995) How multiple-synapse boutons could preserve input specificity during an interneuronal spread of LTP. Trends Neurosci 18:365-369.

Hatton GI (1990) Emerging concepts of structure-function dynamics in adult brain: the hypothalamo-neurohypophysial system. Prog Neurobiol 34:37-504.

Hayes RL, Yang K, Whitson JS, Postmantur R (1995) Cytoskeletal derangements following central nervous system injury: modulation by neurotrophic gene transfection. J Neurotrauma 12:933-941.

Humm JL, Kozlowski DA, James DC, Gotts JE, Schallert T (1998) Use-dependent exacerbation of brain damage occurs during an early post-lesion vulnerable period. Brain Res 783:286-292.

Isackson PJ (1995) Trophic factor response to neuronal stimuli or injury. Curr Opin Neurobiol 5:350-357.

Jones TA (1999) Multiple synapse formation in the motor cortex opposite unilateral sensorimotor cortex lesions in adult rats. J Comp Neurol 414:57-66.

Jones TA, Schallert T (1992a) Overgrowth and pruning of dendrites in adult rats recovering from neocortical damage. Brain Res 581:156-160.

Jones TA, Schallert T (1992b) Subcortical deterioration after cortical damage: effects of diazepam and relation to recovery of function. Behav Brain Res 51:1-13.

Jones TA, Schallert T (1994) Use-dependent growth of pyramidal neurons after neocortical damage. J Neurosci 14:2140-2152.

Jones TA, Kleim JA, Greenough WT (1996) Synaptogenesis and dendritic growth in the cortex opposite unilateral sensorimotor cortex damage in adult rats: A quantitative electron microscopic examination. Brain Res 733:142-148.

Jones TA, Klintsova AY, Kilman VL, Sirevaag AM, Greenough WT (1997) Induction of multiple synapses by experience in the visual cortex of adult rats. Neurobiol Learn Mem 68:13-20.

Jones TA, Hawrylak N, Klintsova AY, Greenough WT (1998) Brain damage, behavior, rehabilitation, recovery, and brain plasticity. Ment Retard Dev Disabil Res Rev 4:231-237.

Kawamata T, Dietrich WD, Schallert T, Gotts JE, Cocke RR, Benowitz LI, Finklestein SP (1997) Intracisternal basic fibroblast growth factor enhances functional recovery and up-regulates the expression of a molecular marker of neuronal sprouting following focal cerebral infarction. Proc Natl Acad Sci USA 94:8179-8184.

Kelley MS, Steward O (1997) Injury-induced physiological events that may modulate gene expression in neurons and glia. Rev Neurosci 8:147-177.

Keller A, Arissian K, Asanuma, H (1992) Synaptic proliferation in the motor cortex of adult cats after long-term thalamic stimulation. J Neurophysiol 68:295-308.

Kleim JA, Lussnig E, Schwarz ER, Comery TA, Greenough WT (1996) Synaptogenesis and Fos expression in the motor cortex of the adult rat after motor skill learning. J Neurosci 16:4529-4535.

Kleim JA, McNamee D, Blankstein EJ, Greenough WT (1997) Interdependent changes in synapse size and number across the course of learning. Soc Neurosci Abstr 23:221.

Kolb B, Gibb R (1991) Environmental enrichment and cortical injury: Behavioral and anatomical consequences of frontal cortex lesions. Cereb Cortex 1:189-198.

Kozlowski DA, James DC, Schallert T (1996) Use-dependent exaggeration of neuronal injury after unilateral sensorimotor cortex lesions. J Neurosci 16:4776-4786.

Nudo RJ, Wise BM, SiFuentes F, Milliken GW (1996) Neural substrates for the effects of rehabilitative training on motor recovery after ischemic infarct. Science 272:1791-1794.

Ridet JL, Malhotra SK, Privat A, Gage FH (1997) Reactive astrocytes: cellular and molecular cues to biological function. Trends Neurosci 20:570-577.

Robinson TE, Kolb B (1997) Persistent structural modifications in nucleus accumbens and prefrontal cortex neurons produced by previous experience with amphetamine. J Neurosci 17:8491-8497.

Rose FD, Davey MJ, Love S, Dell PA (1987) Environmental enrichment and recovery from contralateral sensory neglect in rats with large unilateral neocortical lesions. Behav Brain Res 24:195-202.

Rose FD, al-Khamees K, Davey MJ, Attree EA (1993) Environmental enrichment following brain damage: an aid to recovery or compensation? Behav Brain Res 56:93-100.

Rosenzweig MR, Krech D, Bennett EL, Diamond MC (1962) Effects of 
environmental complexity and training on brain chemistry and anatomy: a replication and extension. J Comp Physiol Psychol 55:429-437.

SAS Institute (1988) SAS/STAT®User's Guide, Release Ed 6.03. Cary, NC: SAS Institute.

Schallert T, Upchurch M, Wilcox RE, Vaughn DM (1983) Posture independent sensorimotor analysis of inter-hemispheric receptor asymmetries in the neostriatum. Pharmacol Biochem Behav 18:753-759.

Schallert T, Whishaw IQ (1984) Bilateral cutaneous stimulation of the somatosensory system in hemidecorticate rats. Behav Neurosci 98:518-540.

Schallert T, Kozlowski DA, Humm JL, Cocke RR (1997) Usedependent structural events in recovery of function. Adv Neurol 73:229-238.

Sheppard AM, Wu JE, Staubli U, Perlmutter LS (1993) Changes in calpain and brain spectrin immunoreactivity accompany sprouting in the deafferented hippocampus. Synapse15:239-42.

Stewart MG, Rusakov DA (1995) Morphological changes associated with stages of memory formation. Behav Brain Res 66:21-28.

Turner AM, Greenough WT (1985) Differential rearing effects on rat visual cortex synapses. I. Synaptic and neuronal density and synapses per neuron. Brain Res 329:195-203.

Wise SP, Donoghue JP (1986) Motor cortex of rodents. In: Cerebral cortex, Vol 5 (Jones EG, Peters A, eds), pp 243-270. New York: Plenum.

Woolley CS, Wenzel HJ, Schwartzkroin PA (1996) Estradiol increases the frequency of multiple synapse boutons in the hippocampal CA1 region of the adult female rat. J Comp Neurol 373:108-117. 